\title{
El arte como instrumento para entender la naturaleza del sufrimiento
}

\author{
Art as an instrument to understand the nature of suffering
}

Continuando con la serie de artículos relacionados con el arte como instrumento educativo en medicina, ${ }^{1}$ nos ocuparemos, en esta oportunidad, de describir la naturaleza del sufrimiento humano, compañero ineludible tanto del enfermo, su familia y sus agentes de salud en particular como de todo el género humano en general. En el presente artículo, exploraremos la naturaleza del sufrimiento a la luz del pensamiento del escritor Marcel Proust, quien se explayó sobre este tema con la autoridad que le confirió la conjugación en él tanto del genio literario como de la condición de enfermo crónico, ya que era un asmático grave., ${ }^{2,3}$

Basaremos, entonces, el presente análisis en dos de sus frases más conocidas respecto del sufrimiento, plasmadas por este autor en los tomos "Albertina ha desaparecido" $\mathrm{y}$ "Sodoma y Gomorra" 5 de su colosal novela En búsqueda del tiempo perdido. Veamos la primera de estas frases: "No se cura un sufrimiento sino a condición de soportarlo plenamente". ${ }^{4}$

Aquí, Proust remite al concepto de que el dolor es inevitable, pues depende de circunstancias externas a nosotros (el dolor es inherente a la existencia misma), mientras que, por el contrario, el sufrimiento es opcional, dado que, en realidad, es un producto de nuestra mente, pues es ella quien posee la potencialidad de disiparlo a través de su reinterpretación, que es el "soportarlo plenamente" al que Proust se refiere. Desde ya que no tiene sentido buscar voluntariamente el dolor, pues eso sería masoquismo, pero, cuando este llega, debe ser aceptado, lo cual no es rendirse (resignación), sino transitarlo con la serenidad necesaria para conseguir su resolución (resignificación). Es decir que, si bien puede haber situaciones dolorosas que se nos impongan, somos siempre dueños de la actitud que adoptaremos frente a ellas (Frankl). ${ }^{6}$ Es el amor fati (amor al destino) nietzscheano o aceptación del dolor que nos ha tocado en suerte para reconvertirlo y evitar que se vuelva sufrimiento (inútil reverberación mental de dicho dolor) y lograr, a la vez, que se transforme en acción en pos de su superación. ${ }^{7}$

Veamos la segunda frase proustiana: "La enfermedad es el médico más escuchado: a la bondad, al saber, no se sabe más que prometer; al sufrimiento se le obedece" ${ }^{\prime}{ }^{5}$

En esta segunda frase, Proust nos transmite la idea de que el dolor es un regalo amargo que nos brinda la vida, pues implica una oportunidad de crecimiento espiritual. El dolor, al mostrarnos nuestra vulnerabilidad, nos saca de la comodidad del espacio personal (ego), del cual difícilmente hubiésemos salido de modo espontáneo, y nos enseña a apreciar el valor de su ausencia (binomio dolor-placer), anula nuestra soberbia, propicia el acercamiento al prójimo (el dolor compartido se aliviana) y nos muestra un camino que, de seguirlo, nos conducirá al crecimiento y la trascendencia. El dolor (físico o espiritual) que no deviene sufrimiento sino entendimiento se convierte en fortaleza (Nietzsche). ${ }^{7}$

El dolor puede llegar a ser motor del desarrollo personal, pues genera la conmoción interior (fractura) indispensable para permitir el remodelado intelectual que nos conduzca al desarrollo espiritual. Porque el dolor incomoda es que brinda un estímulo para el cambio y la transformación superadora, pero es nuestra tarea emprenderla. La comprensión del verdadero sentido del dolor (un hecho real y momentáneo) y su diferencia con el sufrimiento (perpetuación de una construcción mental) es de sumo valor para que el agente de salud adquiera la capacidad para asimilar su sufrimiento personal y pueda ayudar a que sus enfermos asimilen el propio, en una suerte de "alquimia espiritual" que trasforma el plomo (dolor) en oro (crecimiento mental) (Jung). ${ }^{8}$

Concluimos, entonces, que la comprensión de la naturaleza y fin del sufrimiento resulta fundamental para los agentes de salud, tanto en su vida personal como laboral, y que el arte es un valioso recurso para lograr dicho entendimiento.

Dr. Carlos G. Musso y Dra. Paula A. Enz Escuela de Medicina, Instituto Universitario del Hospital Italiano de Buenos Aires, Argentina.

http:/ /dx.doi.org/10.5546/aap.2016.196

\section{REFERENCIAS}

1. Musso CG, Enz PA. El arte como instrumento educativo en medicina. Arch Argent Pediatr 2014;112(6):494-5.

2. Beutler M. Los hombres dela historia. Proust. Buenos Aires: Centro Editor de América Latina; 1986.

3. MussoCG. Obras maestras del arte universal y la medicina: En busca del tiempo perdido de Marcel Proust (1871-1922). Evid Actual Pract Ambul 2012;15(2):58.

4. Proust M. En busca del tiempo perdido: Albertina ha desaparecido. Buenos Aires: CS Ediciones; 2006.

5. Proust M. En busca del tiempo perdido: Sodoma y Gomorra. Buenos Aires: CS Ediciones; 2006.

6. Frankl VE. El hombre en busca de sentido. Barcelona: Herder; 1991.

7. Valesi E. Friedrich Nietzsche. La máscara de Dionisos. Buenos Aires: Lea; 2012.

8. Jung CG. El libro rojo. Buenos Aires: El hilo de Ariadna; 2012. 


\section{Uso y abuso de drogas antiangiogénicas en el tratamiento de la retinopatía del prematuro en Argentina, en 2015 Use and abuse of anti-angiogenic drugs for Retinopathy of Prematurity treatment in Argentina, 2015}

Permítannos comenzar este comentario reproduciendo el título de un artículo publicado en $2012^{1}$ por Graham Quinn (EE. UU.), Brian Darlow (NZ) y Andrea Zin (Br), referentes internacionales en la prevención, el diagnóstico y el tratamiento de la retinopatía del prematuro (retinopathy of prematurity; $\mathrm{ROP}$, por sus siglas en inglés).

El conocimiento de la cascada de factores vasoproliferativos en el desarrollo de la vasculatura retiniana aumentó el interés por el uso de agentes antiangiogénicos en el tratamiento de la ROP grave. La utilización de bevacizumab se ha difundido como uno de los más efectivos.

El estudio aleatorizado, multicéntrico (14 hospitales de Texas, EE. UU.) de Mintz-Hittner y col., ${ }^{2}$ denominado BEAT-ROP, publicado en New Engl J Med en 2011, pese a que la población enrolada fue pequeña (143 niños tratados, 286 ojos), concluyó impulsando el uso de Avastin ${ }^{\circledR}$ (bevacizumab) como tratamiento de elección en la ROP grave en Zona I o agresiva posterior (AP$\mathrm{ROP}),{ }^{3}$ también conocida como rush disease por su rapidez evolutiva al desprendimiento de la retina.

La real eficacia de este tratamiento off label (uso no validado por el fabricante) llevó a la utilización rápidamente ascendente del bevacizumab, sin considerar sus posibles efectos secundarios. El estudio BEAT-ROP no estuvo diseñado para evaluar los efectos oculares y generales colaterales del fármaco utilizado. Tampoco se estudió la concentración sérica del fármaco ni su tiempo de permanencia y de eliminación en el torrente sanguíneo (se han detectado niveles después de 15 días de su administración intraocular).

Debemos recordar que la ROP se desarrolla a una edad gestacional en la que existe un rápido crecimiento de vasos pulmonares, renales y cerebrales, entre otros. Su interrupción por acción directa de los antiangiogénicos podría ocasionar graves secuelas en su evolución a distancia; no lo sabemos.

Pero, para los que hemos convivido con la enfermedad ROP desde la fibroplasia retrolental (FRL) como simples observadores de una ceguera terminal, continuando con la primera clasificación internacional en 1984, que nos permitió ubicarnos en "tiempo y espacio", hasta sus posteriores ablaciones retinianas por crio y laserterapia con resultados y efectos colaterales diversos, la interpretación fisiopatológica presentada en marzo de 2001 por el grupo sueco encabezado por Ann Hellstrom ${ }^{4}$ en Proceedings of the National Academy of Sciences of USA (PNAS) y avalado por Mary Ellen Avery marcó un hito, un antes y un después, y nos permitió conocer los pasos del accionar del factor de crecimiento insulínico tipo 1 (Insulin-like growth factor 1; IGF-1, por sus siglas en inglés) y el factor de crecimiento endotelial vascular (Vascular endothelial growth factor; VEGF, por sus siglas en inglés) en el desarrollo de la retina normal y sus cambios en la ROP.

A partir de entonces, comenzaron las investigaciones terapéuticas centradas en el bloqueo intravítreo de la VEGF con los antiangiogénicos, que estaban en pleno auge como tratamiento del cáncer y sus ramificaciones multiorgánicas. Las diferentes drogas paulatinamente se centraron en el bevacizumab $\mathrm{y}$, específicamente, en el Avastin ${ }^{\circledR}$ como fármaco de elección.

El entusiasmo observado en los oftalmólogos con experiencia en el tratamiento de la ROP fue comprensible y avalado por el menor daño a la retina y la necesidad inferior de repetir el tratamiento varias veces, comparado con la crio o laserterapia.

No obstante, su uso off label restringía su indicación. Finalmente, nuestra Administración Nacional de Medicamentos, Alimentos y Tecnología Médica (ANMAT) aceptó su utilización en situaciones bien definidas: ROP en estadio +3 plus o agresiva posterior, bajo protocolo hospitalario y consentimiento informado de los padres; indicaciones similares a las propuestas por Mintz-Hittner ${ }^{5}$ en 2009.

También su indicación ante fracasos con laserterapia fue ampliamente difundida entre los oftalmólogos pediátricos, como medida humanitaria ante una discapacidad visual grave inminente.

El Grupo ROP Argentina, en la Guía de Práctica Clínica (GPC) para la prevención, el diagnóstico y el tratamiento de la ROP, recientemente publicada, ${ }^{6}$ recomienda: "Considerar la monoterapia intravítrea con bevacizumab (sin necesidad de la fotocoagulación con láser) antes de que se desarrolle el desprendimiento de retina en ROP grado 3 plus en Zona I (no en Zona II), con 
hemorragias, pupila rígida, neovascularización intravítrea (con mínima fibrosis) o agresiva posterior (AP-ROP): dosis de $0,625 \mathrm{mg}$ a través de pars plana a $2 \mathrm{~mm}$ del limbo".

Sin embargo, son infrecuentes los estadios graves de ROP, directamente relacionados con la edad gestacional; en los más inmaduros, su prevalencia es mayor. Si consideramos a todos los niños tratados, la AP-ROP se presenta entre el 5\%$10 \%$ de los casos; dicho de otra manera, 1 cada 1020 prematuros afectados tendrían indicación de antiangiogénicos por aplicación intraocular.

Hasta aquí, su uso se indica en una patología grave que llevaría rápidamente al desprendimiento de la retina y a la ceguera, en la mayoría de los casos.

Pero, al comenzar este comentario, hablábamos del abuso (RAE: uso excesivo, indebido, injusto) de las drogas antiangiogénicas en el tratamiento de la ROP, en Argentina durante 2015.

Observemos, en la Figura 1, los datos aportados por el Grupo ROP Argentina (XIV Jornada Nacional, 37. CONARPE-SAP, Mendoza, 2015) en 88 Servicios de Neonatología y en las 24 provincias argentinas, donde se reportaron 212834 nacimientos en 2014. Sobre 7371 niños con riesgo de desarrollar ROP $(2502<1500 \mathrm{~g}), 1000$ desarrollaron la enfermedad y la cuarta parte requirió tratamiento (265).

Después de un inicio "controlado" en su indicación durante 2011 y 2012, el aumento en el uso del bevacizumab fue sorprendente; en 2014, $18 \%$ recibieron antiangiogénicos como única terapéutica o combinada con laserterapia: casi 1 cada 5 niños tratados.

Preocupa aún más si analizamos la cifra de 2015 (primer semestre): $\mathbf{2 9} \%$ de los casos tratados recibieron bevacizumab, $\mathrm{i} 1$ cada 3 niños!

Realidades como la expresada no son nuevas en medicina; sobran ejemplos de sobreindicaciones

Figura 1. Porcentaje de todos los recién nacidos tratados con bevacizumab (Avastin) sobre el total de niños tratados, cómo único tratamiento o combinado con laserterapia, entre 2011 y 2014. Fuente: Grupo ROP Argentina (con permiso).

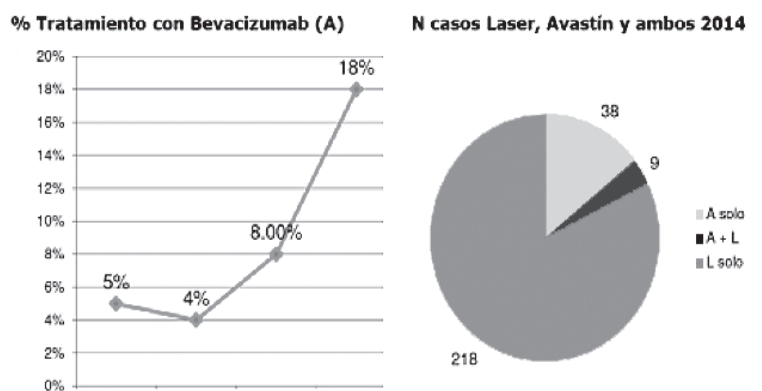

farmacológicas ante resultados iniciales casi "mágicos", sin evidencias que ratifiquen su uso y sus posibles efectos colaterales.

La investigación en terapias con antiangiogénicos para ROP no se ha centrado únicamente en bevacizumab; también hay experiencia con ranibizumab y propanolol. ${ }^{7} \mathrm{La}$ ventaja mayor con este último fármaco es su administración oral y, en un futuro cercano, su administración por gotas oftálmicas, un real desafío terapéutico.

Los antiangiogénicos forman parte de la terapéutica de la ROP y nadie lo puede poner en duda, pero, de cualquier manera, la fotocoagulación con láser sigue siendo el "estándar de oro".

Se requerirán rigurosos estudios clínicos, multicéntricos, controlados y con seguimiento a largo plazo para brindar evidencias que justifiquen su correcta indicación en la terapéutica de una enfermedad con posibilidad de ser invalidante de por vida. ${ }^{8}$ En nuestro estado actual del conocimiento de esta terapia prometedora, la prudencia debe ser prioritaria.

Ernesto R. Alda

Médico pediatra neonatólogo.

Jefe del Servicio de Neonatología, Hospital Privado del Sur, Bahía Blanca. Docente de la carrera de Medicina, Departamento de Ciencias de la Salud, Universidad Nacional del Sur, Bahía Blanca. Miembro del Grupo ROP Argentina

http:/ /dx.doi.org/10.5546/aap.2016.197

\section{REFERENCIAS}

1. Quinn GE, Darlow BA, Zin AA. Use and abuse of anti-angiogenic drugs for ROP treatment. Vision 2020 Latinoamérica Boletín trimestral. Enero 2012. Disponible en https://vision2020la. wordpress.com/traducciones/use-and-abuse-of-anti-angiogenicdrugs-for-rop-treatment/

2. Mintz-Hittner HA, Kennedy KA, Chuang AZ. Efficacy of intravitreal bevacizumab for stage $3+$ retinopathy of prematurity. N Engl J Med 2011;364(7):603-15.

3. Reynolds JD. Bevacizumab for retinopathy of prematurity. NEngl J Med 2011;364(7):677-8.

4. Hellstrom A, Perruzzi C, Ju M, Engstrom E, et al. Low IGF-I suppresses VEGF-survival signaling in retinal endothelial cells: direct correlation with clinical retinopathy of prematurity. Proc Natl Acad Sci USA 2001;98(10):5804-8.

5. Mintz-Hittner HA, Best LM. Antivascular endothelial growth factor for retinopathy of prematurity. Curr Opin Pediatr 2009;21(2):182-7.

6. Grupo ROP Argentina. Ministerio de Salud. Guía de Práctica Clínica para la prevención, diagnóstico y tratamiento de la Retinopatía del Prematuro (ROP). Buenos Aires, 2015. [Acceso: 12 de enero de 2016]. Disponible en: http:/www.msal.gob.ar/images/stories/bes/ graficos/0000000723cnt-guia-pract-clin-ROP-2015.pdf.

7. Filippi L, Cavallaro G, Bagnoli P, Dal Monte M, et al. Oral propranolol for retinopathy of prematurity: risks, safety concerns, and perspectives. J Pediatr 2013;163(6):1570-7.

8. Pertl L, Steinwender G, Mayer C, Hausberger S, et al. A systematic review and meta-analysis on the safety of Vascular Endothelial Growth Factor (VEGF) Inhibitors for the treatment of retinopathy of prematurity. PLoS One 2015;10(6):e0129383. 